\title{
Performance Evaluation of Algorithms for the State of Charge Estimation of Storage Devices in Microgrid Operation
}

\author{
F. Adinolfi, F. Conte, S. Massucco, M. Saviozzi, F. Silvestro \\ DITEN, Università degli Studi di Genova \\ via all'Opera Pia, 11/A \\ I-16145, Genova, Italy \\ stefano.massucco@unige.it
}

\author{
S. Grillo \\ DEIB, Politecnico di Milano \\ p.zza Leonardo da Vinci, 32 \\ I-20133, Milano, Italy \\ samuele.grillo@polimi.it
}

\begin{abstract}
This paper analyzes different Kalman filtering algorithms for the real-time State of Charge (SoC) estimation of Battery Energy Storage System (BESS). Accurate SoC estimation is a key issue for microgrid real-time operation involving optimal model-based control. A BESS composed of Li-ion battery equipped with a Battery Management System (BMS) is characterized by fitting the parameters of a dynamic model, validated through experimental tests. Particular attention is devoted to the identification and representation of model nonlinearities in order to design robust Kalman filtering SoC estimation methods. Performance evaluation of the proposed algorithms are carried out by statistical simulations and experimental real-time tests. The analysis also takes in consideration the computational performances of the different methods in order to match the requirements of real-time control routines.
\end{abstract}

Index Terms-State of charge estimation, storage device, Kalman filter, microgrid.

\section{INTRODUCTION}

In recent years, power systems are experiencing quite a large revolution due to the massive penetration of power generators fed by renewable sources like wind and sun and the concurrent course in power generation practice of using distributed and dispersed medium and small generators. The combination of these two tendencies is essential towards a cleaner environment and also for a more resilience network if properly managed.

The combination of intermittency and diffusion may also bring out and worsen problems in transmission and distribution networks [1]. Using a traditional planning approach the solution can be provided only by transmission and distribution equipment reinforcement, which is, for sure, the most costly way to work out the complex problems posed by this new context. A different solution is offered by managing distribution networks using a microgrid approach. In this context microgrid and in particular storage can perform their full role to guarantee a more flexible network. Therefore, it is necessary integrate energy storage devices in the control loop of these future networks.

Storage systems can be exploited by owners and investors to mitigate the uncertainty deriving from renewable energy randomness. In fact, energy storage can be seen not only as an "energy buffer" to be used to keep the energy delivered at Point of Common Coupling (PCC) as close as possible to the declared value, but also as a real time reservoirs that can react to power imbalances of the grid. This use of storage devices is more challenging since the State of Charge (SoC) has to be computed in real time in order to optimal control the microgrid in a short time interval. In [2] a lithium-ion storage battery is incorporated into the microgrid during gridconnected operation and islanded operation using a Model Predictive Control. These types of approach require a precise SoC estimation to perform optimal decision over the time horizon.

Traditionally, state of sharge (SoC) estimation algorithms are designed and tested mainly on single cells [3], [4], [5], [6]. This commonly-used procedure is suited to test the effectiveness of the different proposed algorithms but has never been implemented on a commercial storage system. In fact, the presence of a battery management system (BMS) cannot be neglected when considering energy storage devices in a power system, especially when used for energy-intensive tasks [7].

Different works on SoC estimation of the single cell have been investigated but for the full control of a microgrid it is essential to perform the estimation of a full Battery Energy Storage System (BESS) including the BMS [8]. An analysis of different SoC estimation algorithms when applied to the scheduling of a storage device is performed. In particular, a Liion battery (made up of 80 , series connected, $40 \mathrm{Ah}, 3.7 \mathrm{~V}$ rated cells), which is operating, as depicted in Fig. 1, in a microgrid at the University of Genova, has been characterized by fitting the parameters of a proper dynamic model.

Several modeling and estimation approaches have been proposed by literature [3], [4], [5], [6], [9], [10], [11]. This paper focuses on Kalman filtering algorithms based on RCequivalent circuit models.

Different Kalman filtering techniques are considered [4], [12], [13]. A statistical analysis is carried out on synthetic data generated by simulating the BESS. Finally on-line communication between SoC estimation routines and BMS is 


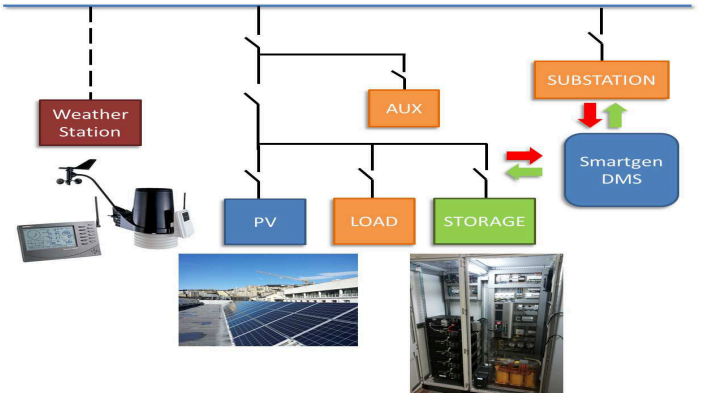

Figure 1. Diagram of the LV microgrid test site at University of Genova.

allowed to test the real-time/real-framework performances of the candidate SoC estimation algorithms.

The paper is organized as follows. Section II describes the BESS characterization. SoC estimation methods are introduced in Section III. Section IV presents the on-line implementation and the experimental results. Finally, conclusions are provided in Section V.

\section{BATTERY CHARACTERIZATION}

\section{A. System Description}

The battery energy storage system (BESS) considered, is a $12 \mathrm{kWh}-10 \mathrm{~kW}$ Lithium-ion battery, composed by five trays connected in series. Each tray includes 16 cells $(3.75 \mathrm{~V}$ and $40 \mathrm{Ah}$ ), thus the rated values of the battery are $300 \mathrm{~V}$ and $40 \mathrm{Ah}$. The energy system available includes also: the switch gear, the inverter, the transformer and the BMS, which interfaces with the operator for managing and controlling the whole system. The BMS provides measures of SoC and both the current flow and voltage value at the DC link bus.

The battery is remotely controlled through a protected Ethernet TCP/IP connection which is established by an OPC Server that collects all the available measurements and commands using Modbus protocol.

\section{B. Model}

The BESS is modelled by a second order equivalent electrical circuit (also called Randles model) [14], [4], [3]. The circuit scheme is depicted in Fig. 2.
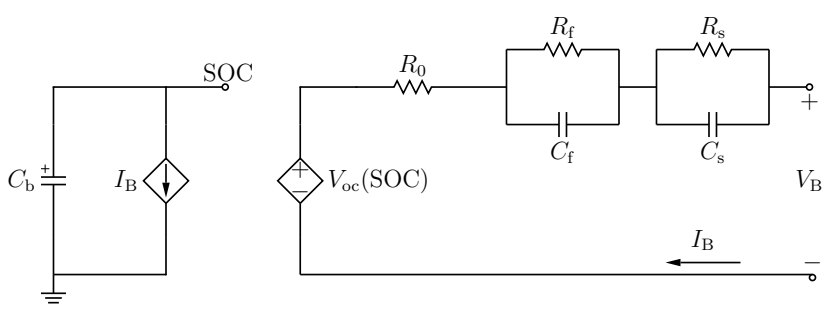

Figure 2. Dynamic model of the energy storage device.

The battery state-of-charge (SoC) [\%] is represented by the voltage $V_{S o C}[\mathrm{~V}]$ across the whole-charge capacitor $C_{b}$ [A]. Battery $\mathrm{SoC}$ and $V_{S o C}$ are related by $\mathrm{SoC}=V_{S o C} / V^{\mathrm{nom}} \cdot 100$, where $V^{\mathrm{nom}}$ is the rated voltage. The model describes the dynamical relation between $V_{S O C}$ and the measured terminal voltage $V_{B}[\mathrm{~V}]$ and load current $I_{B}[\mathrm{~A}]$. Accordingly to Fig. 2, $V_{B}$ is expressed as a function of $V_{S o C}$ and $I_{B}$ :

$$
V_{B}=V_{O C}\left(V_{S o C}\right)-V_{f}-V_{s}-R_{0} I_{B}
$$

where:

- $V_{O C}\left(V_{S o C}\right)$ is the nonlinear mapping from the battery SoC and the open-circuit voltage (OCV) $V_{O C}$ [V] (hereafter referred to as $S o C-O C V$ map);

- $V_{f}$ and $V_{s}[\mathrm{~V}]$ are the voltages across the two RC networks $\left(R_{f}, C_{f}\right)$ and $\left(R_{s}, C_{s}\right)$, respectively;

- $R_{0}[\Omega]$ is the internal battery resistance.

The dynamics of $V_{f}, V_{s}$ and $V_{S o C}$ is given by

$$
\begin{aligned}
\dot{V}_{f} & =-\frac{1}{R_{f} C_{f}} V_{f}+\frac{1}{C_{f}} I_{B} \\
\dot{V}_{s} & =-\frac{1}{R_{s} C_{s}} V_{s}+\frac{1}{C_{s}} I_{B} \\
\dot{V}_{S o C} & =-\frac{1}{C_{b}} I_{B} .
\end{aligned}
$$

\section{Characterization Tests}

Similarly to [3], [4], the identification of the model parameters is based on the test results obtained by driving the battery by load current defined paths. In particular, two profiles (charge and discharge) are designed: starting from a state of total discharge/charge, battery is fully charged/discharged through a sequence of current steps. Each step is 2 minutes long and followed by 12 minutes pause, during which the system rests. The steps amplitude is the nominal current value allowed by the BMS: which is about the declared value of 40 A. Globally the tests last for 9 hours. Both charge and discharge tests are conducted two times. All measurements are collected with $1 \mathrm{sec}$ granularity.

The OCV $V_{O C}$, is assumed equal to the voltage value measured at the end of the resting period. In Fig. 3 the circles show when the $V_{O C}$ evaluation is performed in a charge test.
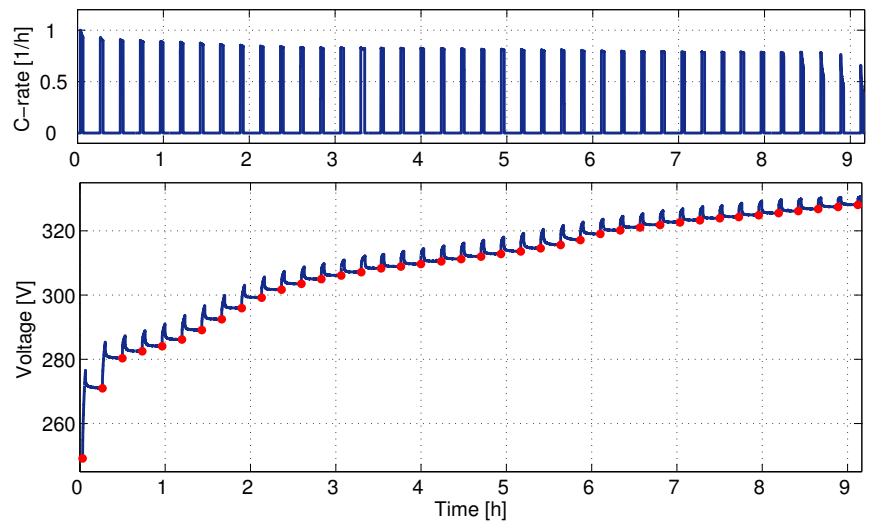

Figure 3. Current and voltage profiles during a charge test.

During the tests each $V_{O C}$ value is collected together with the SoC provided by the BMS. Globally, 140 pairs of $\left(\mathrm{SoC}, V_{O C}\right)$ values are registered and processed through a 
fitting procedure to identify the SoC-OCV map. The best result in terms of minimal root-mean-square-error (RMSE) is obtained adopting a truncated Fourier series fitting function of sixth order with 14 coefficients, depicted in Fig. 4 together with the registered pairs $\left(\mathrm{SoC}, V_{O C}\right)$.

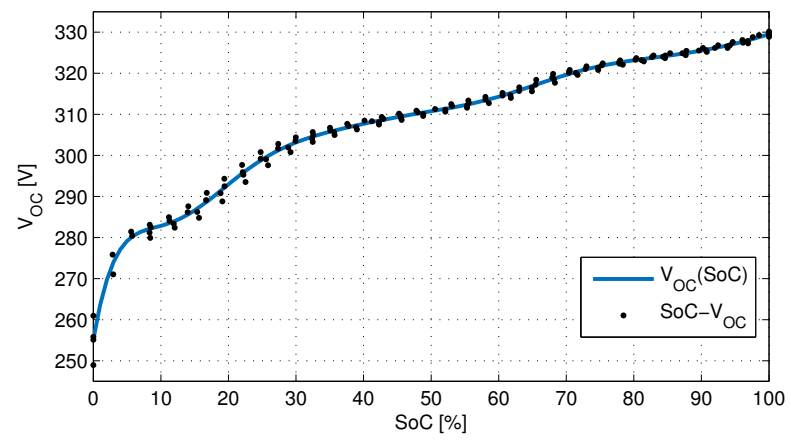

Figure 4. SoC-OCV map truncated Fourier series fitting curve. Black dots are the registered pairs $\left(\mathrm{SoC}, V_{O C}\right)$.

\section{Parameters Identification}

Referring to model (1)-(4) described in section II-B, (1) can be formulated in the Laplace domain as follows:

$$
\begin{gathered}
V_{B}(s)=V_{O C}(s)-R_{0} I_{B}(s)-G_{m}(s) I_{B}(s), \\
G_{m}(s)=\frac{R_{s}}{1+s R_{s} C_{s}}+\frac{R_{f}}{1+s R_{f} C_{f}} .
\end{gathered}
$$

Assuming (5)-(6), parameters are determined as follows.

- $C_{b}$ : excluding the temperature effects, the whole-charge capacity is equal to $3600 \cdot$ (Nominal Capacity) [Ah]. The resultant capacity is therefore $C_{b}=144000 \mathrm{~F}$.

- $R_{0}$ : the internal resistance is evaluated as the ratio between the instantaneous voltage variation and the amplitude of the $k$-th current step as shown in Fig. 5:

$$
R_{0, k}=\frac{v_{\min , k}-v_{\max , k}}{i_{\min , k}-i_{\max , k}} .
$$
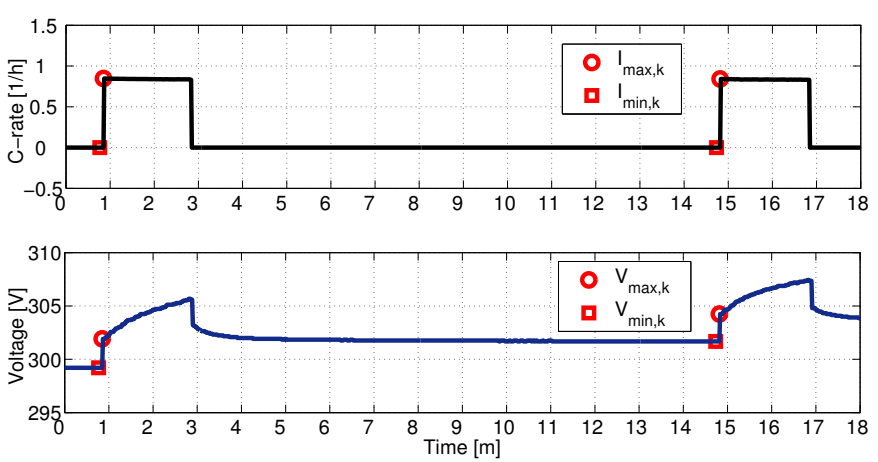

Figure 5. $R_{0, k}$ evaluation example.

The global value of $R_{0}$ is calculated as the mean value of whole set of the computed internal resistances:

$$
R_{0}=\sum_{k=1}^{N} R_{0, k} / N
$$

- $R C$ Network: (5) can be rewritten as

$$
V_{O C}-V_{B}-R_{0} I_{B}=G_{m} I_{B}=: V_{e q},
$$

where, assuming $d I_{B} / d t=0$ in the sampling pe$\operatorname{riod} T_{s}=1 \mathrm{sec}$, time-discretization provides, using $Z$ transform:

$$
\begin{aligned}
V_{e q}(z)= & \frac{R_{f}\left(1-e^{-T_{s} / T_{p f}}\right) z^{-1}}{1-e^{-T_{s} / T_{p f}} z^{-1}} \\
& \left.+\frac{R_{s}\left(1-e^{-T_{s} / T_{p s}}\right) z^{-1}}{1-e^{-T_{s} / T_{p s}} z^{-1}}\right) I_{B}(z) \\
= & \left(\frac{z^{-1}\left(b_{1}+b_{2} z^{-1}\right)}{1+a_{1} z^{-1}+a_{2} z^{-2}}\right) I_{B}(z)
\end{aligned}
$$

where, $T_{p s}, T_{p f}$ are the time constants of the two $R C$ sub-networks. Coefficients $a_{1}, a_{2}, b_{1}, b_{2}$ were identified by the ARX system identification method [12]. These values were then combined to calculate the parameters in Table I.

TABLE I. $\quad R C$ Network Parameters

\begin{tabular}{cc}
\hline \hline Parameter & Value \\
\hline$R_{0}$ & $78.3 \mathrm{~m} \Omega$ \\
$R_{f}$ & $41.2 \mathrm{~m} \Omega$ \\
$R_{s}$ & $35.2 \mathrm{~m} \Omega$ \\
$C_{f}$ & $561.94 \mathrm{~F}$ \\
$C_{s}$ & $4943.08 \mathrm{~F}$ \\
$T_{p f}$ & $23.15 \mathrm{~s}$ \\
$T_{p s}$ & $173.87 \mathrm{~s}$ \\
\hline
\end{tabular}

\section{E. Validation}

The identified model parameters are validated by four simulation tests. The values reported in Section II-D are exploited in model (1)-(4) and used to reply the paths run by the battery during the characterization tests. One of the $V_{B}$ profiles during discharge simulations is graphically reported in Fig. 6, while the Mean Absolute Percentage Errors (MAPE), calculated according to (11), are listed in Table II:

$$
M A P E=\frac{1}{N} \sum_{i=1}^{N}\left|\frac{V_{B, \text { real }, i}-V_{B, \text { sim }, i}}{V_{B, \text { real }, i}}\right| \cdot 100
$$

where: $N$ is the number of samples, $V_{B, r e a l, i}$ is the $i$-th value of the measured voltage, and $V_{B, s i m, i}$ is the $i$-th value of the simulated voltage.

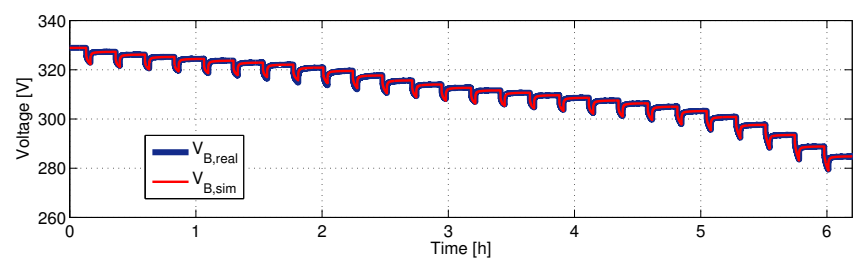

Figure 6. Real and simulated behavior in a discharge tests. 
TABLE II. MAPE VALUES OF VALIDATION TESTS

\begin{tabular}{rc}
\hline \hline Test & $M A P E[\%]$ \\
\hline charge 1 & 0.0334 \\
charge 2 & 0.0471 \\
discharge 1 & 0.0387 \\
discharge 2 & 0.0440 \\
\hline
\end{tabular}

\section{SoC Estimation Methods}

The objective of this work is to consider Kalman filtering procedures to estimate the BESS SoC following an approach similar to [3], [4], [5], [6], but considering a proper linear or nonlinear representation of the BESS SoC-OCV map. The idea is to correct through a filtering algorithm based on the dynamic model (1)-(4) the SoC estimated by Coulomb Counting methodologies, which consist in the simple integration of the differential equation (4). Notice that observer based approaches like the one in [3] have been not considered since BMS measurements are time sampled and therefore require discrete-time estimation methods.

In order to introduce the filtering techniques, in the following Section III-A, model (1)-(4) is firstly written in the standard continuous-time state space form. In Section III-B different representations of the system nonlinearities are discussed. In Section III-C, once time-discretization is operated, model uncertainty and measurements errors are suitably modelled. Finally, Section III-D introduces the filtering procedures and in Section III-E off-line validation tests are presented.

\section{A. State-space representation}

As said in Section II-A, the measurements provided by the BMS, with a sampling time $T_{s}$, are the terminal voltage $V_{B}$ and the load current $I_{B}$. The latter is usually considered as a known control variable but in the case of the complex BESS subject of this paper, to consider current as a measured quantity is more reasonable. Therefore, assuming again $d I_{B} / d t=0$ within a sampling interval, model (1)-(4) can be written in the continuous-time state-space form

$$
\begin{aligned}
& \dot{x}=\bar{A} x \\
& y=h(x)
\end{aligned}
$$

where $x$ and $y$ are the system state and output vector, respectively defined as

$$
x=\left[\begin{array}{llll}
V_{f} & V_{s} & V_{S o C} & I_{B}
\end{array}\right]^{T}, \quad y=\left[\begin{array}{ll}
V_{B} & I_{B}
\end{array}\right]^{T}
$$

and where

$$
\begin{gathered}
\bar{A}=\left[\begin{array}{cccc}
-\frac{1}{R_{f} C_{f}} & 0 & 0 & \frac{1}{C_{f}} \\
0 & -\frac{1}{R_{s} C_{s}} & 0 & \frac{1}{C_{s}} \\
0 & 0 & 0 & -\frac{1}{C_{b}} \\
0 & 0 & 0 & 0
\end{array}\right], \\
h(x)=\left[\begin{array}{c}
V_{O C}\left(x_{3}\right)-x_{1}-x_{2}-R_{0} x_{4} \\
x_{4}
\end{array}\right] .
\end{gathered}
$$

\section{B. Nonlinear modelling of the SoC-OCV map}

System (12)-(13) is nonlinear because of the SoC-OCV map appearing in the output map (16). Therefore, nonlinear Kalman filtering approaches are required. It is well known that nonlinear filtering performances depend on the model accuracy, as well as for any model based estimation algorithm, but also on the capability of representation and/or approximation of the system nonlinearities. To find the optimal tradeoff between accuracy and mathematical simplicity is always needed. Therefore, three possible representation of the SoCOCV map are considered here:

- truncated Fourier series fitting curve: as mentioned in Section II-C it has resulted as the best fitting curve in terms of minimal RMSE among a considerable set of possible candidates. In this case the SoC-OCV map (expressed as function of $V_{S o C}=x_{3}$ ) has the following form:

$$
V_{O C}^{F}\left(x_{3}\right)=a_{0}+\sum_{i=1}^{6}\left[a_{i}\left(\cos \left(i w x_{3}\right)\right)+b_{i}\left(\sin \left(i w x_{3}\right)\right)\right] ;
$$

- polynomial fitting curve: the SoC-OCV map is modelled by a fifth order polynomial function:

$$
V_{O C}^{P}\left(x_{3}\right)=\sum_{i=0}^{5} a_{i} x_{3}^{i}
$$

- linear approximation: as proposed in [4], within the interval $10-100 \%$, the SoC-OCV map is represented by a linear function:

$$
V_{O C}^{L}\left(x_{3}\right)=a_{0}+a_{1} x_{3} .
$$

Figure 7 depicts the versions of the three listed types of fitting curves, each obtained by minimizing the RMSEs reported in Table III. Concerning the linear approximation, optimization has been computed considering the SoC interval $10-100 \%$. Just regarding Fig. 7 , it results clear that the truncated Fourier series is the more accurate, whereas linearization is the poorest, especially within the $\mathrm{SoC}$ interval $0-20 \%$. Accuracy is however paid with complexity. Indeed, the Fourier representation is the most complex and nonlinear.

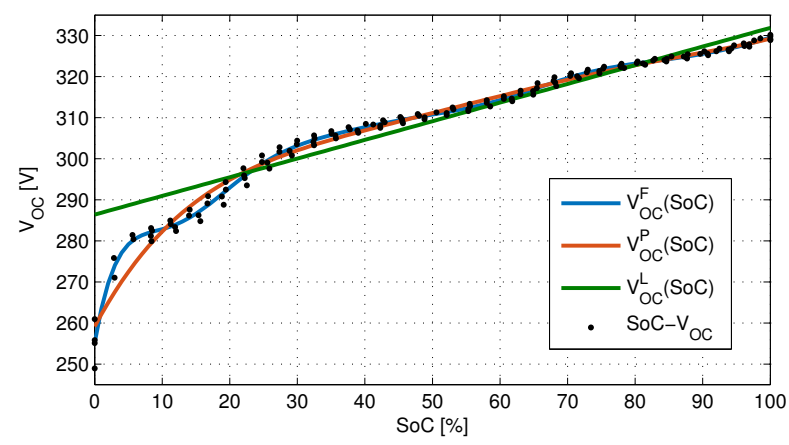

Figure 7. SoC-OCV map representations with truncated Fourier series, polynomial, and linear fittings. Black dots are the registered pairs (SoC, $V_{O C}$ ). 
TABLE III. RMSES OF SOC-OCV MAP FITTING CURVES.

\begin{tabular}{rc}
\hline \hline Fitting curve & RMSE [V] \\
\hline Fourier & 1.2487 \\
Polynomial & 1.4403 \\
Linear & 2.7710 \\
\hline
\end{tabular}

On the contrary, using linearization the output map (16), and consequently the overall system (12)-(13), become linear.

The error committed using any of presented SoC-OCV maps, can be modelled by a zero-mean Gaussian noise term $\varepsilon$, with standard deviation $\sigma_{\varepsilon}$ set equal to the RMSEs reported in Table III. Therefore, $V_{O C}\left(x_{3}\right)$ in (16) is substituted with

$$
V_{O C}\left(x_{3}\right)=V_{O C}^{\mathrm{ty}}\left(x_{3}\right)+\varepsilon^{\mathrm{ty}}
$$

where ty $=\mathrm{F}, \mathrm{P}$, or L, depending on the type of fitting curve.

\section{Time-discretization and uncertainties modelling}

After time-discretization system (12)-(13) assumes the form

$$
\begin{aligned}
x_{k+1} & =A x_{k}+w_{k}, \\
y_{k} & =h\left(x_{k}\right)+v_{k},
\end{aligned}
$$

where $x_{k}=x\left(T_{s} k\right), y_{k}=y\left(T_{s} k\right)$ and $A=e^{\bar{A} T_{s}}$. Sequences $w_{k}$ and $v_{k}$ are supposed to be zero-mean, white, Gaussian and mutually independent. They are added to the state and output equations in order to represent the model uncertainties and the measurement errors. More precisely:

- $w_{k}$ is characterized by the covariance matrix $\Psi_{w}$ and models the state equations uncertainty, i.e. the uncertainty on the parameters of (2)-(4) identified in Section II;

- $v_{k}$ is composed by two independent components, $v_{k}=\left[\begin{array}{ll}v_{1, k} & v_{2, k}\end{array}\right]$. First component $v_{1, k}$ is related to the measurement of the terminal voltage $V_{B}$. It is supposed to be the sum of two independent noises: $\varepsilon_{k}^{\text {ty }}$, representing the uncertainty of the SoC-OCV map (see Section III-B), and $n_{V, k}$, with variance $\sigma_{V}^{2}$, modelling the voltage metering errors. Under these assumptions, $v_{1, k}$ results to be characterized by the variance $\sigma_{\varepsilon}^{2}+\sigma_{V}^{2}$. Second component $v_{2, k}$ models the current meter errors and it is characterized by the variance $\sigma_{I}^{2}$.

\section{Kalman Filtering Methods}

System (21)-(22) is amenable to be processed by a Kalman filtering procedure to estimate the state vector $x_{k}$, whose third component corresponds to the estimate of the SoC voltage $V_{O C}$ at the time step $k$. Taking into account the three types of SoC-OCV map fitting curves presented in Section III-B, three different Kalman filtering procedures, listed in the following, have been selected.

- Kalman filter $(K F)$ : using the linear approximation $V_{O C}^{L}(S o C)$, standard (linear) KF [15] can be applied to the linear system (21)-(22), as done in [4]. In this case no linearization is operated by the filtering procedure. Therefore, filtering optimally manages the model, which, as remarked above, is however less accurate with respect to nonlinear models.

- Extended Kalman Filter (EKF): using $V_{O C}^{F}(S o C)$ or $V_{O C}^{P}(S o C)$ the nonlinear system (21)-(22) is processed by the standard EKF algorithm [15], which operates the linearization of the output map (16) at each filtering step. In this case, model is more accurate with respect to the case of KF, but filtering must approximate $V_{O C}^{F}(S o C)$ or $V_{O C}^{P}(S o C)$ through linearization.

- Unscented Kalman Filter (UKF): using $V_{O C}^{F}(S o C)$ or $V_{O C}^{P}(S o C)$ the nonlinear system (21)-(22) is processed by the UKF algorithm [13], which has represented an interesting novelty in the nonlinear filtering field thanks to the idea of approximating the state conditional probability density function (PDF), instead of the system equations, with a bounded number of parameters. This algorithm has been proved to be superior to EKF with a comparable computational complexity. In this case no direct approximation of $V_{O C}^{F}(S o C)$ or $V_{O C}^{P}(S o C)$ is operated by the filtering procedure.

To summarize, five candidate algorithms are defined: L-KF: KF using $V_{O C}^{L}$; F-EKF: EKF using $V_{O C}^{F}$; P-EKF: EKF using $V_{O C}^{P}$; F-UKF: UKF using $V_{O C}^{F}$; P-UKF: UKF using $V_{O C}^{P}$.

\section{E. Off-line tests}

The proposed filtering algorithms are analyzed through a statistical analysis employing synthetic data. The BESS characterized in Section II-C is simulated by implementing model (1)-(4) on the Matlab platform. This synthetic battery is initialized with $\mathrm{SoC}=85 \%$ and driven by the 12 hours load current profile depicted in Fig. 8.

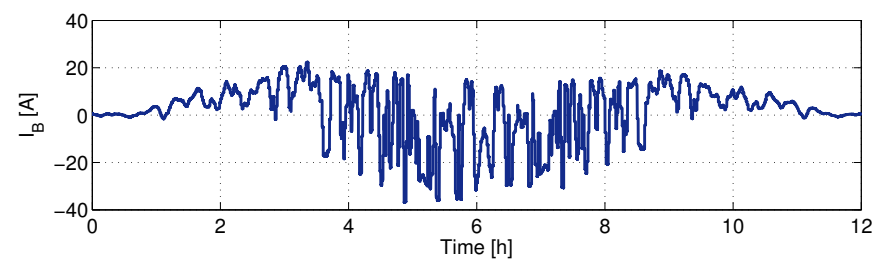

Figure 8. Load current profile used to drive the synthetic battery.

Measurements of $I_{B}$ and $V_{B}$ are generated with sampling time $T_{s}=1 \mathrm{sec}$, corrupted by additive Gaussian noise, with standard deviations $\sigma_{I}=0.1 \mathrm{~A}$ and $\sigma_{V}=100 \mathrm{mV}$, respectively. The measurements of $V_{B}$ are generated by (1) using the Fourier fitting curve in Fig. 4 as "real" SoC-OCV map. Two sets of synthetic data are generated, both consisting of 100 realizations of random noises. In the first set (Dataset 1) no perturbation to the SoC-OCV map is simulated. In the second set (Dataset 2), the SoC-OCV map is perturbed with a Gaussian random noise with variance 1.2487 , i.e. equal to the RMSE computed for the Fourier fitting curve (see Table III).

The synthetic data are processed by the estimation algorithms introduced in Section III-D. All filters start from an initial SoC estimate with $10 \%$ Gaussian error. At each time step, the RMSEs of all SoC estimates are computed over the 
100 noise realizations. Then time average is operated to obtain the performance index values reported in Table IV. Such an index is referred to simply as RMSE.

TABLE IV. SOC ESTIMATION RMSES AND COMPUTATIONAL TIMES.

\begin{tabular}{rccc}
\hline \hline & \multicolumn{2}{c}{ SoC RMSEs [\%] } & \\
\cline { 2 - 3 } Filter & Dataset 1 & Dataset 2 & Comp. Time [ms] \\
\hline KF & 6.53 & 10.78 & 38.0 \\
F-EKF & 0.37 & 2.16 & 87.7 \\
P-EKF & 3.07 & 5.88 & 61.3 \\
F-UKF & 0.12 & 2.02 & 415.0 \\
P-UKF & 3.04 & 5.88 & 320.8 \\
\hline
\end{tabular}

It appears clear that nonlinear filtering is more adequate since using standard KF introduces significant estimation errors. The RMSE is halved by adopting the polynomial representation in P-EKF and P-UKF for both datasets. Errors are further decreased when the Fourier representation is employed. However, this occurs more significantly for dataset 1 . This is not surprising because the Fourier representation is used as "real" SoC-OCV map. In dataset 2, where the SoC-OCV map is perturbed, the relative difference between these two classes of filters is reduced. In general, no significant differences result between UKF and EKF. Figure 9 shows the SoC estimates obtained for one of the noise realizations in Dataset 1.
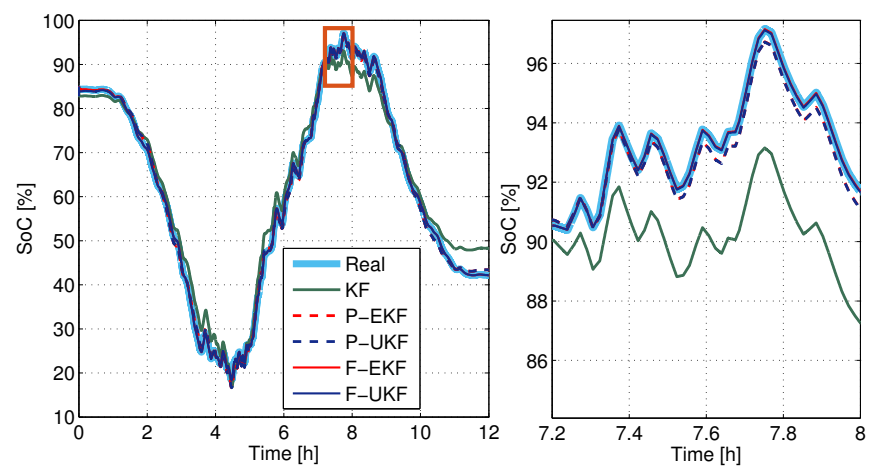

Figure 9. Example of SoC estimation results with no perturbation of the SoC-OCV map. Right figure is a zoom of the section of the SoC profiles within the orange rectangle in the left figure. In both the figures red and blue lines (solid and dashed) are overlapping almost everywhere.

It is important to stress that both EKF and UKF have no problems in managing the battery model nonlinearities. More precisely, no typical drawbacks of nonlinear filters, such as the possible divergence of estimates due to linearization and/or numerical errors, occur in the case under consideration. There are no drawbacks also from the computational point of view. Indeed, the average Matlab computational time per filtering iteration, executed by a CPU Intel(R) Core(TM) i-7 $2.70 \mathrm{GHz}$, is always lower than the $1 \mathrm{sec}$ sampling time, as shown in Table IV. However, the UKF computational burden is significantly higher. Thus, considering that estimation results are comparable, it can be concluded that EKF is preferable to UKF. Finally notice that there are no severe time differences between the use of polynomial and Fourier series fitting curves. Therefore, from the present study there is no motivation to prefer the simplest polynomial representation to the Fourier series one.

\section{ON-LINE IMPLEMENTATION AND EXPERIMENTAL RESULTS}

As described in Section II-A, an OPC Server allows the considered BESS to be remotely monitored. The estimation algorithms introduced in Section III have been implemented on the Matlab(R) platform in order to be executed in real-time and to communicate with the OPC Server, which provides the BMS measurements with $1 \mathrm{sec}$ granularity.

All filters are initialized with the SoC measurements provided by the BMS, assuming Gaussian error with a $10 \%$ standard deviation. Filtering parameters are: $\sigma_{V}=1 \mathrm{mV}, \sigma_{I}=0.1 \mathrm{~A}, \sigma_{\varepsilon}$ equal to the RMSEs in Table III, depending on the considered filter, and

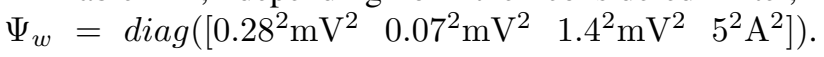

In order to evaluate the performances of the filtering algorithms in this real-time framework, the three SoC battery profiles depicted in Fig. 10 have been generated. In Profile 1, SoC moves within the interval 70-85\%, in Profile 2 between $40-75 \%$, in Profile 3 between 5-25\%. All profiles are obtained by driving the BESS with the load current path reported in the top pictures of Fig. 10 starting from different initial SoC $(\approx 85 \%, \approx 55 \%, \approx 25 \%$, respectively). Such a path consists of twelve sub-profiles of 10 minutes, separated by pause intervals lasting 20 or 30 minutes.

During pauses, $\mathrm{SoC}$ does not change, whereas the measured terminal voltage $V_{B}$ converges to the corresponding OCV. Therefore, a set of thirteen OCV values is collected for each profile by registering the terminal voltages $V_{B}$ at the end of the pause intervals. The real SoC levels reached at the beginning of pauses are then post-computed from the collected OCVs by inverting SoC-OCV map. The inverse map is obtained with high accuracy by employing the spline smoothing fitting technique [16] over the same 40 pairs $\left(\mathrm{SoC}, V_{O C}\right)$ used to identify the direct SoC-OCV maps in III-C.

The filtering performances can be thus evaluated by computing thirteen SoC estimation errors for each profile, as difference between the filters SoC estimates at the beginning of pauses and the thirteen post-computed real SoC values.

Figure 10 shows the estimation results of KF, F-EKF and F-UKF compared with the BMS SoC measurements and the post-computed real SoC values, depicted at the beginning of pause intervals as black squares. P-EKF and P-UKF are not reported in Fig. 10 in order to preserve the figures readability. Bottom figures depict the absolute values of the thirteen SoC estimation errors per profile. It appears clear that F-EKF and F-UKF significantly improve the estimation performances both of KF and BMS, in particular in the $40-75 \%$ (Profile 2) and $5-25 \%$ (Profile 3) SoC zones. Notice that, without surprise, in the $5-25 \%$ zone, $\mathrm{KF}$ is completely inadequate. However, also BMS measurements become largely imprecise, whereas F-EKF and F-UKF seems to keep similar performances is all the considered SoC zones. 

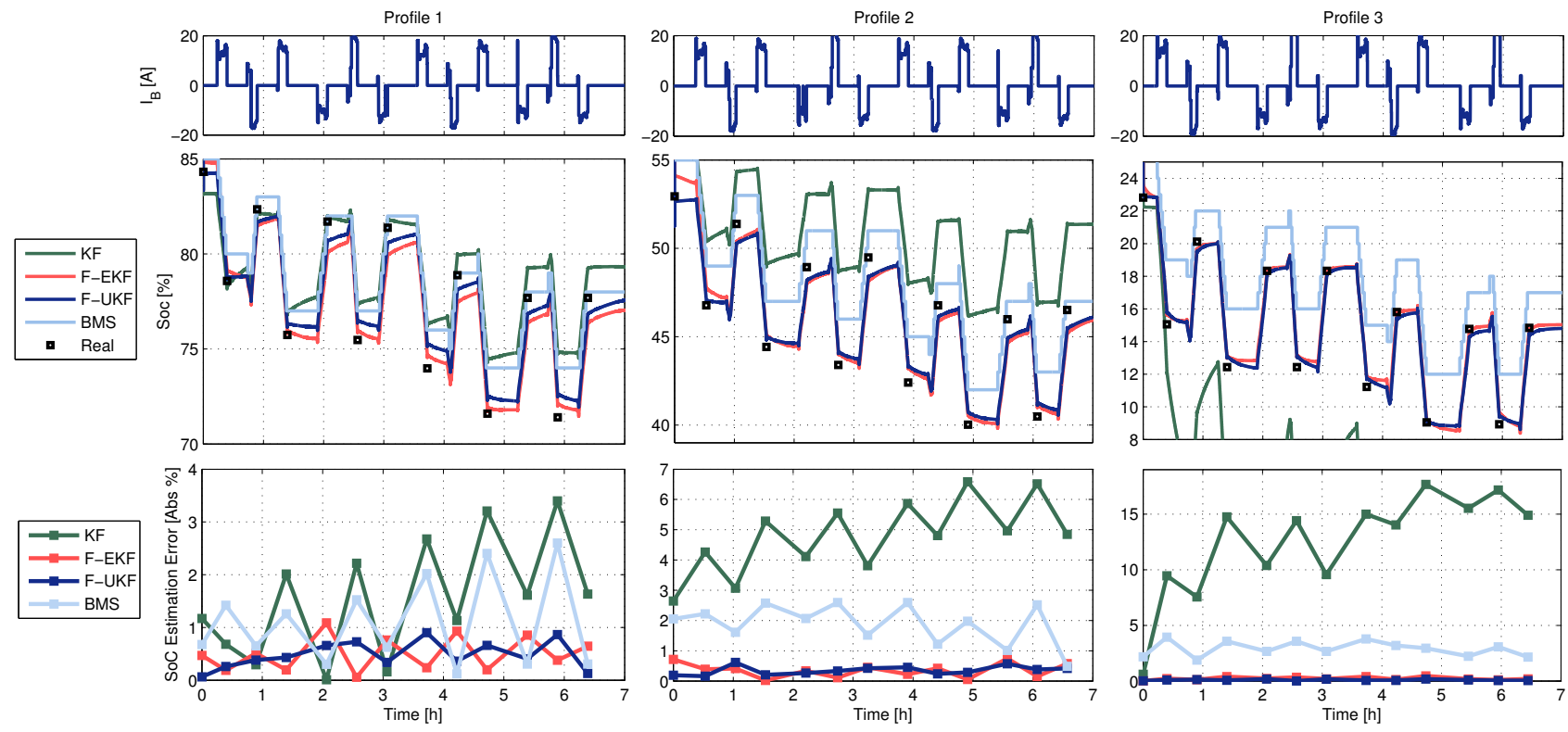

Figure 10. Real-time SoC estimation results. Top figures are the measured load current profiles. Center figures depict the estimated SoC profiles, together with the BMS SoC measurements and the set of post-computed real SoC values. Bottom figures depict the absolute values of the SoC estimation errors in correspondence of the post-computed real SoC values.

TABLE V. EXPERIMENTAL RESULTS: SOC ESTIMATION RMSEs [\%]

\begin{tabular}{rccc}
\hline \hline Filter & Profile 1 & Profile 2 & Profile 3 \\
\hline KF & 1.89 & 4.93 & 13.20 \\
F-EKF & 0.54 & 0.37 & 0.10 \\
P-EKF & 1.44 & 0.99 & 1.57 \\
F-UKF & 0.59 & 0.42 & 0.26 \\
P-UKF & 1.35 & 0.97 & 1.66 \\
BMS & 1.36 & 1.99 & 2.98 \\
\hline
\end{tabular}

These considerations are confirmed by the numerical data in Table V, which provides the RMSEs computed over the SoC estimation errors, including also P-EKF and P-UKF. The F-EKF results to have the better performances, even if comparable with the F-UKF, exactly as it arises in the off-line tests presented in Section III-E. Generally, all results obtained in the off-line tests are confirmed by the experimental data.

The conclusions of the study can be summarized as follows. Standard KF with linear approximation of the SoC-OCV map (proposed for example in [4]) is inadequate for a complex BESS; EKF and UKF have comparable estimation accuracy; EKF is preferable because of a lower computational burden; the truncated Fourier series representation of the SoC-OCV map is preferable to the polynomial one.

\section{CONCLUSIONS}

In this work a full characterization of a real BESS coupled to a PV plant has been carried out. SoC estimation is of paramount importance in power system application. In fact, as the presence of renewable (and, thus, intermittent) energy sources grows, energy management must exploit energy storage devices in order to increase efficiency and allow regulation. Optimal scheduling and management algorithms strongly rely on the estimation of the actual state of the grids.

SoC evolution is nonlinear and it has been demonstrated that the simple integration of the current flowing through the BESS may lead to great errors. A bad estimation of the SoC may impair the correct operation of those algorithms, as may lead to inefficient (or even unfeasible) actions.

In this paper, five different algorithms for on-line $\mathrm{SoC}$ estimation have been tested on a real BESS. They all have been compared to the traditional BMS provided by the manufacturer. Extended Kalman Filter with truncated Fourier series representation of nonlinearities has resulted as the best algorithm both for estimation accuracy and computational time.

Future works will be devoted to the consideration of more complex battery models to evaluate the impact on the estimation performances into the context of microgrid operation.

\section{REFERENCES}

[1] A. Nagarajan and R. Ayyanar, "Design and strategy for the deployment of energy storage systems in a distribution feeder with penetration of renewable resources," IEEE Trans. Sust. Energy, vol. 6, no. 3, pp. 10851092, July 2015.

[2] K. Tan, P. So, Y. Chu, and M. Chen, "Coordinated control and energy management of distributed generation inverters in a microgrid," IEEE Trans. Pow. Syst., vol. 28, no. 2, pp. 704-713, April 2013.

[3] M. Gholizadeh and F. Salmasi, "Estimation of State of Charge, Unknown Nonlinearities, and State of Health of a Lithium-Ion Battery Based on a Comprehensive Unobservable Model," vol. 61, no. 3, pp. 1335-1344, Mar. 2014.

[4] P. Spagnol, S. Rossi, and S. Savaresi, "Kalman Filter SoC estimation for Li-Ion batteries," in IEEE International Conference on Control Applications (CCA), Sep. 2011, pp. 587-592.

[5] G. L. Plett, "Extended Kalman filtering for battery management systems of LiPB-based HEV battery packs: Part 2. Modeling and identification," Journal of Power Sources, vol. 134, no. 2, pp. 262-276, 2004. 
[6] _ "Extended Kalman filtering for battery management systems of LiPB-based HEV battery packs: Part 3. State and parameter estimation," Journal of Power Sources, vol. 134, no. 2, pp. 277-292, 2004.

[7] S. Grillo, M. Marinelli, S. Massucco, and F. Silvestro, "Optimal Management Strategy of a Battery-Based Storage System to Improve Renewable Energy Integration in Distribution Networks," vol. 3, no. 2, pp. 950-958, Jun. 2012.

[8] T. Kim and W. Qiao, "A hybrid battery model capable of capturing dynamic circuit characteristics and nonlinear capacity effects," in IEEE PES General Meeting, July 2012, pp. 1-1.

[9] D. Di Domenico, G. Fiengo, and A. Stefanopoulou, "Lithium-ion battery state of charge estimation with a kalman filter based on a electrochemical model," in IEEE CCA 2008, Sept 2008, pp. 702-707.

[10] F. Sun, X. Hu, Y. Zou, and S. Li, "Adaptive unscented kalman filtering for state of charge estimation of a lithium-ion battery for electric vehicles," Energy, vol. 36, no. 5, pp. 3531-3540, 2011.

[11] W. He, N. Williard, C. Chen, and M. Pecht, "State of charge estimation for electric vehicle batteries using unscented kalman filtering," Microelectronics Reliability, vol. 53, no. 6, pp. 840-847, 2013.

[12] M. Verhaegen and V. Verdult, Filtering and System Identification: A Least Squares Approach. Cambridge University Press, 2007.

[13] S. J. Julier and J. K. Uhlmann, "A new method for the nonlinear transformation of means and covariances in filters and estimators," IEEE Trans. Aut. Contr., vol. 45, no. 3, pp. 477-482, Mar. 2000.

[14] M. Chen and G. Rincon-Mora, "Accurate electrical battery model capable of predicting runtime and i-v performance," vol. 21 , no. 2 , pp. 504-511, June 2006.

[15] B. Anderson and J. Moore, Optimal filtering. Prentice-Hall, Inc., 1979.

[16] T. J. Hastie and R. Tibshirani, Generalized Additive Models. Chapman and Hall, 1990. 